\title{
Evaluation of diet pattern and weight gain in postmenopausal women enrolled in the Women's Health Initiative Observational Study
}

\author{
Christopher Ford ${ }^{1}$, Shine Chang $^{2}$, Mara Z. Vitolins ${ }^{3}$, Jenifer I. Fenton ${ }^{4}$, Barbara V. Howard ${ }^{5}$, Jinnie J. Rhee ${ }^{6}$, \\ Marcia Stefanick ${ }^{7}$, Bertha Chen ${ }^{8}$, Linda Snetselaar ${ }^{9}$, Rachel Urrutia ${ }^{10}$ and Alexis C. Frazier-Wood ${ }^{11_{*}}$ \\ ${ }^{1}$ Emory Global Diabetes Research Center, Emory University, Atlanta, GA 30322, USA \\ ${ }^{2}$ Department of Epidemiology, University of Texas MD Anderson Cancer Center, Houston, TX 77030, USA \\ ${ }^{3}$ Department of Epidemiology and Prevention, Wake Forest School of Medicine, Winston-Salem, NC 27101, USA \\ ${ }^{4}$ Department of Food Science and Human Nutrition, Michigan State University, East Lansing, MI 48824, USA \\ ${ }^{5}$ Center for the Study of Sex Differences in Health, Aging and Disease, MedStar Health Research Institute and Georgetown/Howard \\ Universities Center for Clinical and Translational Research, Washington, DC 20057, USA \\ ${ }^{6}$ Division of Nephrology, Stanford School of Medicine, Stanford, CA 94304, USA \\ ${ }^{7}$ Stanford School of Medicine, Stanford Prevention Research Center, Stanford, CA 94305, USA \\ ${ }^{8}$ Department of Obstetrics and Gynecology, Stanford School of Medicine, Stanford, CA 94305, USA \\ ${ }^{9}$ Department of Epidemiology, University of Iowa College of Public Health, Iowa City, IA 52246, USA \\ ${ }^{10}$ Department of Obstetrics and Gynecology, University of North Carolina at Chapel Hill School of Medicine, Chapel Hill, NC 27516, USA \\ ${ }^{11}$ Children's Nutrition Research Center, Baylor College of Medicine, Room CNRC-2036, Mail Stop BCM320, Houston, TX 77030, USA
}

(Submitted 19 July 2016 - Final revision received 24 February 2017 - Accepted 27 March 2017- First published online 16 May 2017)

\section{Abstract}

It is unclear which of four popular contemporary diet patterns is best for weight maintenance among postmenopausal women. Four dietary patterns were characterised among postmenopausal women aged 49-81 years (mean 63.6 (sD 7.4) years) from the Women's Health Initiative Observational Study: (1) a low-fat diet; (2) a reduced-carbohydrate diet; (3) a Mediterranean-style (Med) diet; and (4) a diet consistent with the US Department of Agriculture's Dietary Guidelines for Americans (DGA). Discrete-time hazards models were used to compare the risk of weight gain $(\geq 10 \%)$ among high adherers of each diet pattern. In adjusted models, the reduced-carbohydrate diet was inversely related to weight gain (OR 0.71; $95 \%$ CI 0.66, 0.76), whereas the low-fat (OR 1.43; $95 \%$ CI 1.33, 1.54) and DGA (OR 1.24; 95\% CI 1.15, 1.33) diets were associated with increased risk of weight gain. By baseline weight status, the reduced-carbohydrate diet was inversely related to weight gain among women who were normal weight (OR 0.72; $95 \%$ CI 0.63, 0.81), overweight (OR 0.67; $95 \%$ CI 0.59, 0.76) or obese class I (OR 0.63; $95 \%$ CI $0.53,0.76)$ at baseline. The low-fat diet was associated with increased risk of weight gain in women who were normal weight (OR 1.28; $95 \%$ CI 1.13, 1.46), overweight (OR 1.60; $95 \%$ CI 1.40, 1.83), obese class I (OR 1.73; $95 \%$ CI 1.43, 2.09) or obese class II (OR 1.44; $95 \%$ CI $1.08,1.92)$ at baseline. These findings suggest that a low-fat diet may promote weight gain, whereas a reduced-carbohydrate diet may decrease risk of postmenopausal weight gain.

Key words: Weight gain: Diets: Obesity prevention: Mediterranean diet: Low-fat diets: Reduced-carbohydrate diets: Postmenopausal women

Many women gain weight during menopause ${ }^{(1,2)}$, which can increase the risk of obesity and related chronic diseases such as diabetes, cancer and $\mathrm{CVD}^{(3,4)}$. Identifying one or more diet patterns that may prevent weight gain could reduce the burden of obesity and related diseases among women in this age group. Although the US Department of Agriculture (USDA) issues the Dietary Guidelines for Americans (DGA) every 5 years, a number of conflicting dietary patterns continue to be investigated for their ability to induce weight-loss ${ }^{(5-9)}$. Despite their popularity, diets such as a Mediterranean-style diet, a low-fat diet and a reduced-carbohydrate diet, have not been compared with the USDA DGA for their role in prevention of weight gain in freeliving postmenopausal women. Moreover, in this area of research, where the majority of studies aim to achieve an energetic deficit, how diet influences weight maintenance when individuals are not asked to reduce their energy intake is largely unexplored. Thus, it remains unclear what overall dietary advice should be provided to this population for the maintenance of weight.

In this study, the relationship between four common diet patterns and weight gain in a heterogeneous sample of US

Abbreviations: 2010-HEI, 2010 Healthy Eating Index; aMed, Alternate Mediterranean Diet; DGA, Dietary Guidelines for Americans.

* Corresponding author: A. C. Frazier-Wood, fax +1 713798 7055, email LekkiWood@Gmail.com 
postmenopausal women was examined in order to inform population-level dietary guidelines for the prevention of weight gain among free-living postmenopausal women in the USA. Using data from the Women's Health Initiative Observational Study (WHI/OS), four diet patterns were characterised: (1) a lowfat diet; (2) a reduced-carbohydrate diet; (3) a Mediterraneanstyle (Med) diet; and (4) a diet consistent with the USDA's DGA. In separate models, hazard ratios were computed by comparing the risk of weight gain in high and low adherers of each diet pattern. Overall hazards by diet pattern and stratified hazards by category of baseline weight status were computed.

\section{Methods}

Sample

Data were included from women who participated in the WHI/ OS, a longitudinal study of postmenopausal women aged 49-81 years who were enrolled between 1994 and 1998, and followed for up to 8 years ( $n$ 93676). Details regarding the sample and design of WHI/OS have been published elsewhere ${ }^{(10)}$. Respondents with a $\mathrm{BMI}<18.5 \mathrm{~kg} / \mathrm{m}^{2}$ ( $n$ 1107), or those who reported following a diabetic diet at baseline ( $n$ 3764), were excluded, leaving 88805 respondents in the final analytic sample. All procedures were conducted in accordance with the Declaration of Helsinki. This study (no. PA16-0039) is exempt from approval by internal review board (reviewed by University of Texas MD Anderson Cancer Center Office of Human Research Ethics).

\section{Outcome}

Height and weight were measured at baseline to classify respondents as normal weight (BMI: $18.5-24.9 \mathrm{~kg} / \mathrm{m}^{2}$ ), overweight (BMI: $25 \cdot 0-29 \cdot 9 \mathrm{~kg} / \mathrm{m}^{2}$ ), obese class I (BMI: $30 \cdot 0$ $34.9 \mathrm{~kg} / \mathrm{m}^{2}$ ), obese class II (BMI: $35.0-39 \cdot 9 \mathrm{~kg} / \mathrm{m}^{2}$ ) or obese class III or more (BMI $\geq 40 \cdot 0 \mathrm{~kg} / \mathrm{m}^{2}$ ). Respondents' self-reported highest weight since last follow-up, assessed at years 1, 3, 4, 5, 6, 7 and 8, was used to compute weight change from baseline. Sensitivity analyses were conducted to examine the correlation between measured weight at baseline, and highest reported weight in the time since last follow-up at year 1 (Pearson's $r$ : 0.87; $P<0 \cdot 001)$. Participants were identified as having experienced the 'outcome' if their reported highest weight since last follow-up was $\geq 10 \%$ higher than baseline weight. In sensitivity analyses comparing 3, 5 and $10 \%$ weight gain, and the average BMI at baseline $\left(27.4 \mathrm{~kg} / \mathrm{m}^{2}\right)$, a $10 \%$ increase in weight was found to be the smallest increment to shift the average BMI to the obese range $\left(30 \cdot 1 \mathrm{~kg} / \mathrm{m}^{2}\right)$. Thus, $\geq 10 \%$ was the threshold used to define the outcome, which was modeled as a binary variable to accommodate a time-to-event analysis. Respondents were censored after developing the outcome, or when lost to follow-up. A sensitivity analysis was also performed using continuous weight change as the outcome of interest.

\section{Dietary data}

At baseline and year 3, dietary data were ascertained using a FFQ comprising 112 items. Dietary intake data from the baseline FFQ was used to assign respondents to a diet pattern. Food, beverage and nutrient intake was computed utilising the University of Minnesota Nutrition Coding Center nutrient database ${ }^{(11,12)}$. The 2010 Healthy Eating Index (2010-HEI) ${ }^{(13)}$ (available from http://appliedresearch.cancer.gov/hei/tools. html), and the MyPyramid Equivalents Database 2.0 $0^{(14)}$, were used to characterise adherence to the DGA diet. Baseline total 2010-HEI scores and component scores were computed for total vegetables; dark green vegetables, peas and beans; total fruit; whole fruit; whole grains; total dairy products; seafood and plant proteins; fatty acids; $\mathrm{Na}$; and refined grains. The Alternate Mediterranean Diet (aMed) score was used to evaluate adherence to a Mediterranean-style diet ${ }^{(15)}$. In brief, the aMed assigns 1 point for each of the following categories if intake is above the sample median: (1) vegetables; (2) legumes; (3) fruit; (4) nuts; (5) whole grains; (6) fish; and (7) ratio of monounsaturated fat:saturated fat. Before computing aMed component scores, intakes were adjusted for total energy, and thus component scores were based on the resulting 'relative' sample medians. In addition, 1 point is given if intake of total red and processed meats is below the median, or if alcohol (ethanol) intake is in the range of $5-25 \mathrm{~g} / \mathrm{d}^{(15)}$. The aMed gives a score of 0-9, which we rescaled to a 100-point scale for congruence with the 2010-HEI. For the 2010-HEI and aMed, a higher score indicates greater adherence with the DGA diet and the Mediterranean-style diet patterns, respectively. Quintile of total score was used to delineate high (top quintile) and low adherers (bottom quintile) of the Mediterranean-style and the DGA diet patterns. Below, we use 'DGA diet' to refer to those in the highest quintile of 2010-HEI score, and 'Mediterranean-style diet' to reference those in the highest quintile of aMed score. Quintiles of 'percentage of total energy from fat' and 'percentage of total energy from carbohydrates' were used to delineate high and low in the low-fat diet and the reduced-carbohydrate diet, respectively. Accordingly, 'low-fat diet' and 'reduced-carbohydrate diet' are used below to refer to those in the lowest quintile of intake fat and carbohydrates, respectively. To accommodate their continuous nature, the four diet patterns were compared using estimates from separate models (one for each diet pattern), rather than from a single combined model.

\section{Covariates}

Sociodemographic information was collected at baseline using a standard questionnaire. This information included annual family income, race/ethnicity (American Indian or Alaskan Native, Asian or Pacific Islander, Black or African American, Hispanic/Latino, White (not of Hispanic Origin), or other), age and highest education level completed. Alcohol intake was assessed by self-report at baseline, with possible responses ranging from 'none to $<1 /$ month' to ' $\geq 3$ each day'. Lifetime smoking status at the time of survey was also ascertained at baseline (current, former and never). Physical activity was assessed at baseline using a standard questionnaire previously shown to have acceptable validity and reliability ${ }^{(16-18)}$. Mild activity was defined as walking. Moderate activity was defined as 'not exhausting' and included biking outdoors, callisthenics, 
easy swimming and dancing. Strenuous or very hard exercise was defined as activities during which 'You work up a sweat and your heart beats fast'. Waist circumference was measured at baseline using a standard protocol.

\section{Statistical analysis}

All analyses were conducted in SAS (version 9.4; SAS Institute Inc.) and Stata (version 14; StataCorp). Discrete-time hazards models were used to model the relationship between diet and weight gain. This approach is appropriate for estimating the hazard when the time to event is represented by a small number of wide intervals such that there are a preponderance of individuals with tied event times ${ }^{(19)}$.

In separate models, the hazard for $\geq 10 \%$ weight gain from baseline was compared among quintiles of a single dietary pattern of interest. All adjusted models controlled for baseline total energy intake (continuous) in order to adjust for potential measurement error in the ascertainment of dietary variables ${ }^{(20)}$. In addition, adjusted models included diet type at year 3 to control for instability of diet class and associated measurement error over time, as well as the following potential confounders $^{(8,15,21-26)}$ : (1) age (continuous); (2) baseline total mild, moderate and hard physical activity as metabolic equivalents of task (MET)-h/week (three continuous variables); (3) race/ ethnicity; (4) annual family income; and (5) baseline smoking status. All categorical variables were modeled using disjoint indicator variables. Completes case analysis was used, whereby respondents with missing data for one or more covariates were excluded from the analyses.

\section{Sensitivity analyses}

We repeated our unadjusted and adjusted analyses specifying the hazard for weight gain from baseline to be $\geq 5 \%$.

\section{Results}

Sample characteristics are given in Table 1 for the eligible sample ( $n$ 88 805) and by level of weight gain at last follow-up. In all, $11 \%$ of respondents ( $n 10109$ ) were missing data for one more covariates. At baseline, women were aged 49-81 years (mean: 63.6 (SD 7.4) years). Respondents were followed an average of 6.9 (SD 1.8), during which 19.5\% ( $n$ 17290) of the sample gained $\geq 10 \%$ of baseline weight. Degree of weight gain was significantly related to age, baseline weight status, waist circumference, education level, household income level, race/ethnicity, weekly MET-h of mild physical activity, smoking status and alcohol use $(P<0 \cdot 01)$. In addition, baseline total energy intake, 2010-HEI score, aMed score, percent of total energy intake from fat, and percent of total energy from carbohydrates, were related to degree of weight gain over time $(P<0 \cdot 01)$.

Selected dietary characteristics for high adherers of each dietary pattern are shown in Table 2 . The Mediterranean-style diet was highest in energy content $(7870 \mathrm{~kJ} / \mathrm{d}(1881 \mathrm{kcal} / \mathrm{d}))$, followed closely by the reduced-carbohydrate diet $(7251 \mathrm{~kJ} / \mathrm{d}$
$(1733 \mathrm{kcal} / \mathrm{d}))$. The low-fat diet was characterised by low dietary fat intake (32 (SD 14) g/d), low dietary cholesterol $(132(\mathrm{SD} 72) \mathrm{mg} / \mathrm{d}$ ) and moderate intake of total dietary fibre $(18(\mathrm{sD} 8) \mathrm{g} / \mathrm{d})$. The reduced-carbohydrate diet was characterised by low intake of carbohydrates (163 (sD 86) g/d), high intake of total fat $(79(\mathrm{SD} 47) \mathrm{g} / \mathrm{d})$ and high intake of dietary cholesterol (299 (sD 199) mg/d). The Mediterranean-style diet was highest in carbohydrate intake $(258$ (SD 86) g/d), total grains (6 (sD 3) servings/d) and alcohol intake (6 (SD 9) servings/ week). The DGA diet was low in fat intake (42 (sD 20) g/d), moderate in carbohydrate intake $(205$ (SD 71) g/d) and highest in intake of total fibre (19 (sD 7) g/d). Macronutrient composition and mean total energy intake among high adherers of each diet pattern is given in Fig. 1. The proportion of total energy from carbohydrates was highest in the low-fat diet (61\%), whereas the proportion of total energy from fat was highest in the reduced-carbohydrate diet (41\%).

\section{Pooled models}

Risk of weight gain among high adherers of each diet was compared with that of low adherers in Table 3. In unadjusted models, high adherence to the low-fat (OR 0.86; $95 \%$ CI 0.82, 0.91 ), Mediterranean-style (OR 0.68; $95 \%$ CI $0.64,0.73$ ) and DGA (OR 0.77; 95\% CI 0.73, 0.81) diets was associated with decreased risk of weight gain. High adherence to the reducedcarbohydrate diet was weakly associated with increased risk of weight gain in unadjusted models (OR 1.05; 95\% CI 1.00, 1.11; $P<0.05)$. In adjusted models, high adherence to the low-fat (OR 1.43; $95 \%$ CI 1.33, 1.54) and DGA (OR 1.24; $95 \%$ CI 1.15, 1.33 ) diets was associated with increased risk of weight gain. There was no longer a significant relationship between diet pattern and risk of weight gain among high adherers to the Mediterranean-style diet (OR 0.95 ; $95 \%$ CI $0.88,1.03$ ) in adjusted models. However, high adherence to the reducedcarbohydrate diet was associated with a sharply lower risk of weight gain in adjusted models (OR 0.71; $95 \%$ CI 0.66, 0.76).

\section{Stratified models}

Baseline weight status was found to be a significant $(P<0 \cdot 10)$ modifier of the relationship between diet pattern and weight gain. Pooled models therefore included an interaction term for baseline weight with diet pattern to obtain a unified estimate of the odds ratios across categories of baseline weight status. The results of these models are shown in Table 4. High adherence to the low-fat diet was associated with increased risk of weight gain among women who were normal weight (OR 1.28; $95 \%$ CI 1.13, 1.46), overweight (OR 1.60; 95\% CI 1.40, 1.83), obese class I (OR 1.73; $95 \%$ CI 1.43, 2.09) or obese class II (OR 1.44; $95 \%$ CI $1.08,1.92)$ at baseline.

High adherence to the reduced-carbohydrate diet was associated with decreased risk of postmenopausal weight gain among women who were normal weight (OR 0.72; 95\% CI 0.63, $0 \cdot 81$ ), overweight (OR $0.67 ; 95 \%$ CI $0.59,0.76)$ or obese class I (OR 0.63; 95\% CI 0.53, 0.76) at baseline.

Across all categories of baseline weight status, high adherence to the Mediterranean-style diet was not significantly 
Table 1. Baseline characteristics of women who participated in the Women's Health Initiative Observational Study according to category of self-reported weight gain during the study ${ }^{\star}$ (Numbers and percentages; mean values and standard deviations)

\begin{tabular}{|c|c|c|c|c|c|c|c|c|c|c|c|c|c|}
\hline & \multirow{2}{*}{\multicolumn{2}{|c|}{ Full sample }} & \multicolumn{2}{|c|}{ Lost $\geq 5 \%$} & \multicolumn{2}{|c|}{$\begin{array}{l}\text { Maintained weight } \\
\text { within } 5 \%\end{array}$} & \multicolumn{2}{|c|}{ Gained $5-10 \%$} & \multicolumn{2}{|c|}{$\begin{array}{c}\text { Gained } \geq 10 \% \text { of } \\
\text { baseline weight }\end{array}$} & \multicolumn{2}{|c|}{ Missing } & \multirow[b]{2}{*}{$P \dagger$} \\
\hline & & & $n$ & $\%$ & $n$ & $\%$ & $n$ & $\%$ & $n$ & $\%$ & $n$ & $\%$ & \\
\hline$n$ & \multicolumn{2}{|c|}{88805} & 1616 & 1.8 & 42512 & 47.9 & 25063 & $28 \cdot 2$ & 17290 & 19.5 & 2324 & $2 \cdot 6$ & \\
\hline Weight status & \multirow{2}{*}{\multicolumn{2}{|c|}{35994}} & & & & & & & & & & & $<0.001$ \\
\hline Normal weight (BMI: $18.5-24.9 \mathrm{~kg} / \mathrm{m}^{2}$ ) & & & 234 & 0.7 & 17396 & $48 \cdot 3$ & 10570 & 29.4 & 7234 & 20.1 & 560 & 1.6 & $<0.001$ \\
\hline Overweight (BMI: $25 \cdot 0-29 \cdot 9 \mathrm{~kg} / \mathrm{m}^{2}$ ) & \multicolumn{2}{|c|}{30316} & 333 & 1.1 & 14709 & 48.5 & 8743 & 28.8 & 5958 & 19.7 & 573 & 1.9 & \\
\hline Obese class I (BMI: $\left.30 \cdot 0-34.9 \mathrm{~kg} / \mathrm{m}^{2}\right)$ & & 259 & 1.9 & 6754 & 49.6 & 3622 & $26 \cdot 6$ & 2556 & $18 \cdot 8$ & 425 & 3.1 & \\
\hline Obese class II (BMI: $35 \cdot 0-39 \cdot 9 \mathrm{~kg} / \mathrm{m}^{2}$ ) & \multicolumn{2}{|c|}{4920} & 130 & 2.6 & 2271 & $46 \cdot 2$ & 1351 & 27.5 & 982 & 20.0 & 186 & 3.8 & \\
\hline Obese class III $\left(\mathrm{BMI} \geq 40.0 \mathrm{~kg} / \mathrm{m}^{2}\right)$ & \multicolumn{2}{|c|}{2902} & 272 & 9.4 & 1288 & 44.4 & 715 & 24.6 & 512 & $17 \cdot 6$ & 115 & 4.0 & \\
\hline Waist circumference $(\mathrm{cm})$ & \multirow{3}{*}{\multicolumn{2}{|c|}{$\begin{array}{l}58915 \\
29518\end{array}$}} & & & & & & & & & & & $<0.001$ \\
\hline$<88$ & & & 959 & 1.6 & 28056 & 47.6 & 17064 & 29.0 & 11648 & $19 \cdot 8$ & 1188 & $2 \cdot 0$ & \\
\hline$\geq 88$ & 29518 & & 633 & $2 \cdot 1$ & 14336 & 48.6 & 7928 & 26.9 & 5593 & 19.0 & 1028 & 3.5 & \\
\hline Highest education completed & \multirow{2}{*}{\multicolumn{2}{|c|}{18657}} & & & & & & & & & & & $<0.001$ \\
\hline Less than high school & & & 360 & 1.9 & 8645 & $46 \cdot 3$ & 5217 & 28.0 & 3684 & $19 \cdot 8$ & 751 & 4.0 & \\
\hline High school diploma or equivalent & \multicolumn{2}{|c|}{32123} & 568 & 1.8 & 15239 & 47.4 & 9015 & 28.1 & 6501 & $20 \cdot 2$ & 800 & 2.5 & \\
\hline Some college & \multicolumn{2}{|c|}{10196} & 182 & 1.8 & 5085 & 49.9 & 2937 & 28.8 & 1780 & 17.5 & 212 & $2 \cdot 1$ & \\
\hline Baccalaureate degree or more & & & 485 & 1.8 & 13212 & 48.8 & 7689 & 28.4 & 5182 & 19.1 & 529 & 2.0 & \\
\hline Household income & & & & & & & & & & & & & $<0.001$ \\
\hline$<\$ 20000$ & & & 307 & 2.4 & 5830 & $45 \cdot 7$ & 3350 & $26 \cdot 2$ & 2642 & 20.7 & 643 & $5 \cdot 0$ & \\
\hline$\$ 20000-\$ 49999$ & & & 620 & 1.7 & 17181 & $48 \cdot 1$ & 10090 & 28.3 & 6999 & $19 \cdot 6$ & 819 & $2 \cdot 3$ & \\
\hline$\$ 50000-\$ 99999$ & & & 398 & 1.6 & 11688 & $47 \cdot 3$ & 7207 & $29 \cdot 2$ & 4974 & 20.2 & 422 & 1.7 & \\
\hline$\geq \$ 100000$ & & & 147 & 1.6 & 4538 & 49.7 & 2665 & 29.2 & 1636 & 17.9 & 141 & 1.5 & \\
\hline Race/ethnicity & & & & & & & & & & & & & $<0.001$ \\
\hline Non-Hispanic White & & & 1257 & 1.7 & 36240 & 48.6 & 21156 & 28.4 & 14404 & $19 \cdot 3$ & 1459 & $2 \cdot 0$ & \\
\hline Non-Hispanic Black & & & 192 & 2.8 & 2943 & $42 \cdot 7$ & 1815 & 26.4 & 1471 & 21.4 & 468 & 6.8 & \\
\hline Hispanic & & & 90 & 2.7 & 1434 & 42.7 & 884 & $26 \cdot 3$ & 684 & 20.4 & 270 & 8.0 & \\
\hline Other & & & 45 & 1.6 & 1322 & 47.6 & 859 & 30.9 & 477 & $17 \cdot 2$ & 77 & $2 \cdot 8$ & \\
\hline Smoking status & & & & & & & & & & & & & $<0.001$ \\
\hline Never & & & 841 & 1.9 & 21760 & 48.9 & 12779 & 28.7 & 7996 & 18.0 & 1132 & 2.5 & \\
\hline Former & & & 620 & 1.7 & 18133 & $48 \cdot 2$ & 10628 & 28.2 & 7354 & 19.5 & 895 & 2.4 & \\
\hline Current & & & 128 & $2 \cdot 4$ & 2020 & 37.4 & 1339 & 24.8 & 1695 & 31.4 & 219 & $4 \cdot 1$ & \\
\hline Alcohol use & & & & & & & & & & & & & $<0.001$ \\
\hline Non-drinker & & & 220 & 2.3 & 4578 & $47 \cdot 8$ & 2629 & 27.5 & 1739 & $18 \cdot 2$ & 411 & $4 \cdot 3$ & \\
\hline Past drinker & & & 330 & 2.1 & 6946 & 43.9 & 4313 & 27.3 & 3629 & 23.0 & 591 & 3.7 & \\
\hline$<1$ drink/month & & & 171 & 1.7 & 4589 & $45 \cdot 0$ & 2947 & 28.9 & 2254 & $22 \cdot 1$ & 237 & $2 \cdot 3$ & \\
\hline$<1$ drink/week & & & 302 & 1.7 & 8425 & 46.9 & 5209 & 29.0 & 3633 & 20.2 & 413 & $2 \cdot 3$ & \\
\hline 1-6 drinks/week & & & 302 & 1.7 & 8425 & 46.9 & 5209 & 29.0 & 3633 & 20.2 & 413 & $2 \cdot 3$ & \\
\hline$\geq 7$ drinks/week & & & 370 & 1.6 & 11605 & 50.1 & 6607 & 28.5 & 4189 & $18 \cdot 1$ & 416 & 1.8 & \\
\hline & Mean & SD & Mean & SD & Mean & SD & Mean & SD & Mean & SD & & & \\
\hline Age & 63.6 & 7.4 & 64.8 & 7.6 & 64.9 & $7 \cdot 2$ & 62.9 & $7 \cdot 2$ & $61 \cdot 0$ & $7 \cdot 2$ & 0 & 0.0 & $<0.001$ \\
\hline Physical activity (MET-h/week) & & & & & & & & & & & & & \\
\hline Mild exercise & 1.4 & $3 \cdot 1$ & 1.3 & 3.1 & 1.4 & $3 \cdot 2$ & 1.4 & 3.1 & $1 \cdot 2$ & 3.0 & 1006 & 1.1 & $<0.001$ \\
\hline Moderate exercise & 3.3 & 5.4 & 3.0 & 5.0 & 3.4 & 5.4 & 3.3 & 5.3 & 3.3 & 5.5 & 1006 & 1.1 & 0.327 \\
\hline Hard exercise & 3.9 & 8.5 & 3.9 & 9.0 & 4.1 & 8.6 & 3.9 & 8.4 & 3.9 & 8.6 & 1006 & 1.1 & 0.160 \\
\hline Diet & & & & & & & & & & & & & \\
\hline Energy intake (kJ/d) & 6486.5 & 2881.5 & 6338.8 & 3233.4 & $6452 \cdot 1$ & 2753.9 & 6544.2 & $2880 \cdot 7$ & $6466 \cdot 4$ & 2997 & 381 & & 0.003 \\
\hline Energy intake (kcal/d) & $1550 \cdot 3$ & 688.7 & $1515 \cdot 0$ & $772 \cdot 8$ & $1542 \cdot 1$ & $658 \cdot 2$ & $1564 \cdot 1$ & 688.5 & 1545.5 & $716 \cdot 3$ & 91 & 0.1 & \\
\hline 2010 Healthy Eating Index score (out of 100 ) & 69.0 & 9.6 & 68.2 & 10.1 & 69.3 & 9.5 & 69.1 & 9.5 & 68.6 & 9.7 & 91 & 0.1 & $<0.001$ \\
\hline Alternate Mediterranean Diet score (out of 9 ) & 4.3 & 1.8 & $4 \cdot 1$ & 1.8 & 4.3 & 1.8 & $4 \cdot 3$ & 1.7 & 4.2 & 1.7 & 91 & $0 \cdot 1$ & $<0.001$ \\
\hline
\end{tabular}

MET, metabolic equivalents of task.

complete data for all covariates of interest.

$+P$ corresponds to a $x^{2}$ test for categorical variables, and ANOVA ( $F$ test) for continuous variables. 
Table 2. Selected dietary characteristics among high adherers of a low-fat, reduced-carbohydrate, Mediterranean-style or Dietary Guidelines for Americans (DGA) diet pattern among women who participated in the Women's Health Initiative Observational Study*

(Mean values and standard deviations)

\begin{tabular}{|c|c|c|c|c|c|c|c|c|}
\hline \multirow[b]{2}{*}{ Total energy intake (kJ/d) } & \multicolumn{2}{|c|}{ Low-fat diet } & \multicolumn{2}{|c|}{ Reduced-carbohydrate diet } & \multicolumn{2}{|c|}{ Mediterranean-style diet } & \multicolumn{2}{|c|}{ DGA diet } \\
\hline & 5770 & 2117 & 7251 & 3673 & 7870 & 3008 & 6012 & 2092 \\
\hline Total energy intake (kcal/d) & 1379 & 506 & 1733 & 878 & 1881 & 719 & 1437 & 500 \\
\hline Carbohydrates (g/d) & 211 & 80 & 163 & 86 & 258 & 86 & 205 & 71 \\
\hline Added sugar (teaspoon equivalents) & 8 & 6 & 8 & 6 & 10 & 6 & 8 & 5 \\
\hline Total dietary fat $(\mathrm{g} / \mathrm{d})$ & 32 & 14 & 79 & 47 & 59 & 36 & 42 & 20 \\
\hline Saturated fat $(\mathrm{g} / \mathrm{d})$ & 10 & 5 & 27 & 17 & 18 & 12 & 13 & 6 \\
\hline Monounsaturated fat $(\mathrm{g} / \mathrm{d})$ & 12 & 5 & 30 & 18 & 23 & 14 & 16 & 8 \\
\hline Polyunsaturated fat (g/d) & 7 & 3 & 16 & 10 & 13 & 8 & 10 & 5 \\
\hline Trans-fat (g/d) & 2 & 1 & 6 & 4 & 4 & 3 & 3 & 2 \\
\hline Protein $(\mathrm{g} / \mathrm{d})$ & 60 & 25 & 74 & 40 & 79 & 32 & 64 & 25 \\
\hline Total dietary fibre $(\mathrm{g} / \mathrm{d})$ & 18 & 8 & 12 & 6 & 24 & 7 & 19 & 7 \\
\hline Soluble fibre $(\mathrm{g} / \mathrm{d})$ & 5 & 2 & 3 & 2 & 6 & 2 & 5 & 2 \\
\hline Insoluble fibre $(\mathrm{g} / \mathrm{d})$ & 13 & 6 & 9 & 5 & 18 & 5 & 14 & 5 \\
\hline Dietary cholesterol (mg/d) & 132 & 72 & 299 & 199 & 200 & 138 & 153 & 85 \\
\hline Servings of fruit & 3 & 1 & 1 & 1 & 3 & 1 & 3 & 1 \\
\hline Servings of vegetables & 3 & 1 & 2 & 1 & 3 & 1 & 3 & 1 \\
\hline Total grains (ounce equivalents) & 5 & 3 & 4 & 3 & 6 & 3 & 4 & 2 \\
\hline Whole grains & 1 & 1 & 1 & 1 & 2 & 1 & 2 & 1 \\
\hline Non-whole grains & 3 & 2 & 4 & 3 & 4 & 2 & 3 & 1 \\
\hline Alcohol intake (servings/week) & 3 & 6 & 6 & 9 & 4 & 4 & 2 & 3 \\
\hline
\end{tabular}

* High adherers to the low-fat and reduced-carbohydrate diet patterns were those in the bottom quintile of percentage of total energy intake from the nutrient of interest, whereas high adherers to the Mediterranean-style and DGA diets were those in the top quintile for Alternate Mediterranean Diet score and 2010 Healthy Eating Index score, respectively.

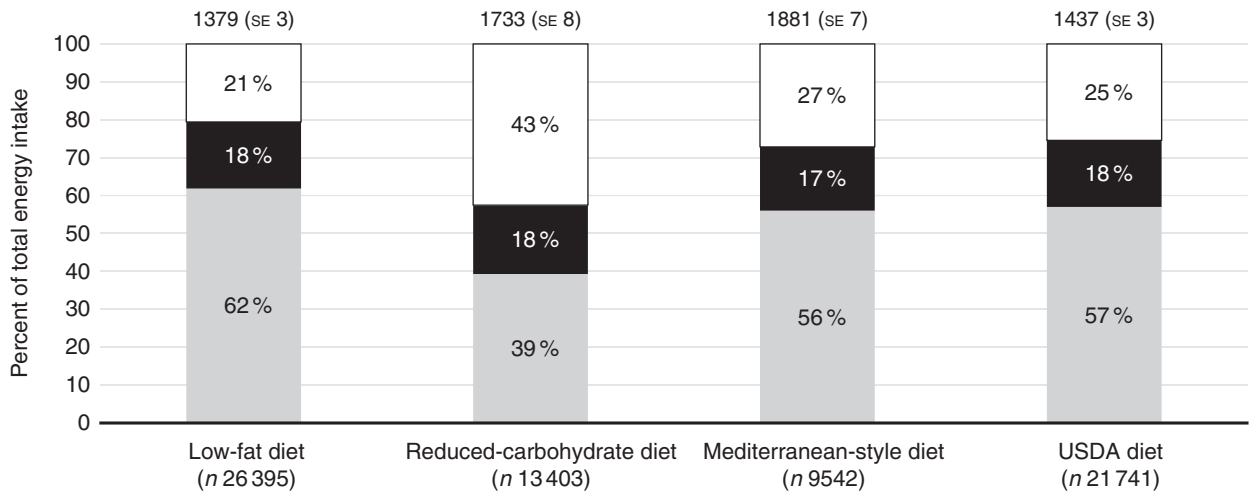

Fig. 1. Total energy intake and percentage of total energy from carbohydrates $(\square)$, fat $(\square)$ and protein ( $\square$ ) among high adherers of a low-fat diet, a reducedcarbohydrate diet, a Mediterranean-style diet and a diet consistent with the US Department of Agriculture's (USDA) Dietary Guidelines for Americans. Total energy intake is given as mean values with their standard errors. Percentages given represent the percent of mean total energy intake. Data are from the Women's Health Observational Study.

related to risk of weight gain, although the relationship approached significance among women who were normal weight at baseline (OR 0.90, 95\% CI 0.90, 1.01; $P=0 \cdot 083$ ).

Conversely, high adherence to the DGA diet was associated with increased risk of weight gain in women who were normal weight (OR 1.13; 95\% CI 1.00, 1.28; $P=0.049$ ), overweight (OR.089; 95\% CI 1.15, 1.48), obese class I (OR 1.41; 95\% CI 1.17, $1.70)$ and obese class III (OR 1.86; $95 \%$ CI 1.18, 2.95). The relationship approached significance among women who were obese class II at baseline (OR 1.33; $95 \%$ CI 0.99, 1.80; $P=0.059$ ).

In sensitivity analyses, a $\geq 5 \%$ weight gain (as opposed to $\geq 10 \%$ weight gain) was used as the primary outcome. The pattern and directionality of the findings were similar to those of the primary analyses with only one exception. In our adjusted model, the relationship between the low-fat diet pattern and weight gain was in the opposite direction of our primary analysis (OR 0.85; $95 \%$ CI 0.80, 0.90).

\section{Discussion}

Overall, we found that postmenopausal women with high adherence to a reduced-carbohydrate diet, with moderate fat and high protein intake, were at decreased risk for postmenopausal weight gain. This finding is consistent with prior related works. Gardner et $a l .{ }^{(27)}$ found that free-living overweight/obese women who consumed reduced-carbohydrate $34.5 \%$ of total energy intake at 12 months) had significantly greater weight loss than those who with higher intake of carbohydrates (range: $45 \cdot 4-52 \cdot 4 \%$ of total energy at 12 months). Moreover, those consuming a low-fat diet $(29.8 \%$ of total energy intake at 12 months) lost significantly less weight than those consuming diets with higher intakes of fat ${ }^{(27)}$. Similarly, Shai et al. ${ }^{(5)}$ found that, with unrestricted energy intake, respondents aged 40-65 years with obesity who followed a low-carbohydrate diet exhibited greater weight loss than those who followed a low-fat 
Table 3. Relative odds of weight gain ( $\geq 10 \%$ from baseline weight $v$. $<10 \%)$ by quintile (Q) of adherence to a low-fat, reducedcarbohydrate, Mediterranean-style or Dietary Guidelines for Americans (DGA) diet pattern among postmenopausal women who participated in the Women's Health Initiative Observational Study* (Odds ratios and $95 \%$ confidence intervals)

\begin{tabular}{|c|c|c|c|c|}
\hline & \multicolumn{2}{|c|}{ Unadjusted ( $n$ 88714) } & \multicolumn{2}{|c|}{ Adjusted (n 70177$)$} \\
\hline & OR & $95 \% \mathrm{Cl}$ & OR & $95 \% \mathrm{Cl}$ \\
\hline \multicolumn{5}{|l|}{ Low-fat diet } \\
\hline Q1 (high adherence) & 0.86 & $0.82,0.91$ & 1.43 & $1.33,1.54$ \\
\hline Q2 & 0.77 & $0.73,0.81$ & $1 \cdot 14$ & $1.06,1.22$ \\
\hline Q3 & 0.79 & $0.74,0.84$ & 1.05 & $0.97,1.13$ \\
\hline Q5 & 0.83 & $0.78,0.89$ & 0.99 & $0.93,1.07$ \\
\hline Q5 (low adherence) & 1.00 & Ref. & 1.00 & Ref. \\
\hline $\begin{array}{l}\quad P_{\text {trend }} \dagger \\
\text { Reduced-carbohydrate diet }\end{array}$ & \multicolumn{2}{|c|}{$<0.001$} & \multicolumn{2}{|c|}{$<0.001$} \\
\hline Q1 (high adherence) & 1.05 & $1.00,1.11$ & 0.71 & $0.66,0.76$ \\
\hline Q2 & 0.92 & $0.88,0.97$ & 0.71 & $0.67,0.76$ \\
\hline Q3 & 0.89 & $0.85,0.93$ & 0.77 & $0.73,0.82$ \\
\hline Q5 & 0.91 & $0.87,0.95$ & 0.84 & $0.80,0.88$ \\
\hline Q5 (low adherence) & 1.00 & Ref. & 1.00 & Ref. \\
\hline$P_{\text {trena }} \dagger$ & \multirow{2}{*}{\multicolumn{2}{|c|}{0.516}} & \multirow{2}{*}{\multicolumn{2}{|c|}{$<0.001$}} \\
\hline Mediterranean-style diet & & & & \\
\hline Q1 (low adherence) & 1.00 & Ref. & 1.00 & Ref. \\
\hline Q2 & 0.89 & $0.85,0.93$ & 0.99 & $0.94,1.05$ \\
\hline Q3 & 0.85 & $0.81,0.89$ & 1.01 & $0.96,1.07$ \\
\hline Q5 & 0.78 & $0.74,0.82$ & 1.00 & $0.94,1.07$ \\
\hline Q5 (high adherence) & 0.68 & $0.64,0.73$ & 0.95 & $0.88,1.03$ \\
\hline $\begin{array}{l}P_{\text {trend }} \dagger \\
\text { DGA diet }\end{array}$ & \multicolumn{2}{|c|}{$<0.001$} & \multicolumn{2}{|c|}{0.513} \\
\hline Q1 (low adherence) & 1.00 & Ref. & 1.00 & Ref. \\
\hline Q2 & 0.89 & $0.84,0.94$ & 1.04 & $0.97,1.11$ \\
\hline Q3 & 0.82 & $0.78,0.87$ & 1.07 & $1.00,1 \cdot 14$ \\
\hline Q5 & 0.80 & $0.76,0.84$ & $1 \cdot 14$ & $1.07,1.22$ \\
\hline Q5 (high adherence) & 0.77 & $0.73,0.81$ & 1.24 & $1.15,1.33$ \\
\hline$P_{\text {trend }} \dagger$ & \multicolumn{2}{|c|}{$<0.001$} & \multicolumn{2}{|c|}{$<0.001$} \\
\hline
\end{tabular}

Ref., referent values.

* All adjusted models controlled for baseline total energy intake (continuous), diet pattern at year 3 of follow-up, age (continuous), baseline total mild, moderate and hard physical activity as metabolic equivalents of task-h/week, race/ethnicity, annual family income and baseline smoking status. All categorical variables (race/ethnicity, annual family income and baseline smoking status) were modeled using disjoint indicator variables.

$\dagger P_{\text {trend }}$ corresponds to a Wald test statistic when a linear term for quintile of diet pattern was substituted in the model.

or Mediterranean diet. In each of these studies, respondents consuming the reduced-carbohydrate and low-fat diet patterns had similar macronutrient intake profiles to the respondents in our study with high adherence to the reduced-carbohydrate and low-fat diets, respectively.

Whereas the reduced-carbohydrate diet was protective against weight gain overall, greater adherence to a low-fat diet was associated with markedly increase of postmenopausal weight gain. This relationship persisted in stratified models (by weight status), wherein high adherence to the low-fat diet pattern was associated with greater risk of weight gain in women who were normal weight to obese class II at baseline. The relationship between the low-fat diet and weight gain was also positive among those with class III obesity at baseline, but did not reach statistical significance. This result stands in contrast to findings from longterm ( $\geq 2$ years) weight loss trials, in which a low-fat diet has been reported to facilitate weight loss ${ }^{(5,28,29)}$. Nonetheless, weight loss trials differ from our study in two important ways that may invalidate comparisons between the two. Foremost, our sample was heterogeneous with the majority of individuals classified as normal weight or overweight by BMI, whereas weight loss trials typically comprise predominantly individuals with obesity ${ }^{(5,28,29)}$. Moreover, achieving an energetic deficit is commonly the goal of weight loss trials, whereas the aim of the current study was to examine the relationship between diet and incident weight gain independent of energetic intake.

Despite these differences, we observed a hierarchical relationship with weight gain among the low-fat, Mediterranean-style and reduced-carbohydrate diets that is consistent with findings from the weight loss trial literature. Shai et al. ${ }^{(5)}$, who compared 2-year weight loss among adults with moderate obesity randomised to a Mediterranean, low-fat, or low-carbohydrate diet, reported that the low-carbohydrate diet was associated with the greatest weight loss, followed by the Mediterranean diet and the low-fat diet (low carbohydrate $>$ Mediterranean $>$ low fat). Similarly, we observed OR of $0.62,1.24$ and 2.05 for the reducedcarbohydrate, Mediterranean-style and low-fat diets, respectively, thereby indicating a hierarchical structure consistent with that reported by Shai et $a l .{ }^{(5)}$.

We also found that regardless of diet pattern they followed, postmenopausal women with a BMI $\geq 35.0 \mathrm{~kg} / \mathrm{m}^{2}$ gained $\geq 10 \%$ of their baseline weight. Although prior studies in adults have found those who were overweight or obese at baseline were more likely to gain weight than those who were normal weight at baseline ${ }^{(30-32)}$, we are unaware of any prior study of weight change over time among adult women in which researchers 
Table 4. Relative odds of weight gain ( $\geq 10 \%$ from baseline weight vs. $<10 \%)$ by baseline weight status and quintile $(Q)$ of adherence to a low-fat, reduced-carbohydrate, Mediterranean-style or Dietary Guidelines for Americans (DGA) diet pattern in postmenopausal women who participated in the Women's Health Initiative Observational Study*

(Odds ratios and $95 \%$ confidence intervals)

\begin{tabular}{|c|c|c|c|c|c|c|c|c|c|c|}
\hline & \multicolumn{2}{|c|}{ Normal weight } & \multicolumn{2}{|c|}{ Overweight } & \multicolumn{2}{|c|}{ Obese class I } & \multicolumn{2}{|c|}{ Obese class II } & \multicolumn{2}{|c|}{ Obese class III } \\
\hline & OR & $95 \% \mathrm{Cl}$ & OR & $95 \% \mathrm{Cl}$ & OR & $95 \% \mathrm{Cl}$ & OR & $95 \% \mathrm{Cl}$ & OR & $95 \% \mathrm{Cl}$ \\
\hline \multicolumn{11}{|l|}{ Low-fat diet } \\
\hline Q1 (high adherence) & 1.28 & $1.13,1.46$ & 1.60 & $1.40,1.83$ & 1.73 & $1.43,2.09$ & 1.44 & $1.08,1.92$ & 1.09 & $0.72,1.65$ \\
\hline Q2 & 1.02 & $0.90,1.15$ & 1.22 & $1.07,1.39$ & 1.34 & $1.12,1.59$ & 1.35 & $1.04,1.75$ & 0.93 & $0.64,1.35$ \\
\hline Q3 & 0.94 & $0.82,1.07$ & $1 \cdot 19$ & $1.04,1.36$ & 1.23 & $1.02,1.48$ & 1.07 & $0.81,1.40$ & 0.81 & $0.56,1.17$ \\
\hline Q5 & 0.89 & $0.78,1.02$ & 1.09 & $0.95,1.25$ & $1 \cdot 10$ & $0.92,1.31$ & 1.06 & $0.82,1.39$ & 0.76 & $0.53,1.09$ \\
\hline Q5 (low adherence) & 1.00 & Ref. & 1.00 & Ref. & 1.00 & Ref. & 1.00 & Ref. & 1.00 & Ref. \\
\hline $\begin{array}{l}\quad P_{\text {trend }} \dagger \\
\text { Reduced-carbohydrate diet }\end{array}$ & \multicolumn{2}{|c|}{$<0.001$} & \multicolumn{2}{|c|}{$<0.001$} & \multicolumn{2}{|c|}{$<0.001$} & \multicolumn{2}{|c|}{0.005} & \multicolumn{2}{|c|}{0.727} \\
\hline Q1 (high adherence) & 0.72 & $0.63,0.81$ & 0.67 & $0.59,0.76$ & 0.63 & $0.53,0.76$ & 0.79 & $0.60,1.05$ & 0.97 & $0.65,1.46$ \\
\hline Q2 & 0.72 & $0.65,0.81$ & 0.73 & $0.65,0.82$ & 0.59 & $0.49,0.70$ & 0.83 & $0.63,1 \cdot 11$ & 0.65 & $0.43,0.99$ \\
\hline Q3 & 0.75 & $0.68,0.83$ & 0.74 & $0.66,0.82$ & 0.78 & $0.67,0.91$ & 1.05 & $0.81,1.37$ & 0.99 & $0.66,1.49$ \\
\hline Q5 & 0.82 & $0.76,0.89$ & 0.81 & $0.74,0.89$ & 0.86 & $0.75,0.99$ & 1.05 & $0.83,1.33$ & 0.98 & $0.67,1.43$ \\
\hline Q5 (low adherence) & 1.00 & Ref. & 1.00 & Ref. & 1.00 & Ref. & 1.00 & Ref. & 1.00 & Ref. \\
\hline $\begin{array}{c}P_{\text {trend }} \dagger \\
\text { Mediterranean-style diet }\end{array}$ & \multicolumn{2}{|c|}{$<0.001$} & \multicolumn{2}{|c|}{$<0.001$} & \multicolumn{2}{|c|}{$<0.001$} & \multicolumn{2}{|c|}{0.040} & \multicolumn{2}{|c|}{0.416} \\
\hline Q1 (low adherence) & 1.00 & Ref. & 1.00 & Ref. & 1.00 & Ref. & 1.00 & Ref. & 1.00 & Ref. \\
\hline Q2 & 0.91 & $0.83,0.99$ & 1.02 & $0.93,1 \cdot 12$ & 1.06 & $0.93,1.22$ & $1 \cdot 16$ & $0.94,1.43$ & 0.79 & $0.57,1.10$ \\
\hline Q3 & 0.99 & $0.90,1.08$ & 0.98 & $0.89,1.08$ & 1.05 & $0.90,1.21$ & $1 \cdot 11$ & $0.88,1.41$ & 1.19 & $0.85,1.67$ \\
\hline Q4 & 0.93 & $0.84,1.03$ & 1.05 & $0.94,1.18$ & 1.09 & $0.92,1.29$ & 0.92 & $0.69,1.25$ & $1 \cdot 12$ & $0.73,1.72$ \\
\hline Q5 (high adherence) & 0.90 & $0.80,1.01$ & 0.95 & $0.83,1.09$ & 1.15 & $0.93,1.42$ & 0.86 & $0.60,1.23$ & 1.08 & $0.61,1.90$ \\
\hline $\begin{array}{l}P_{\text {trend }} \dagger \\
\text { DGA diet }\end{array}$ & \multicolumn{2}{|c|}{0.159} & \multicolumn{2}{|c|}{0.831} & \multicolumn{2}{|c|}{0.206} & \multicolumn{2}{|c|}{0.531} & \multicolumn{2}{|c|}{0.403} \\
\hline Q1 (low adherence) & 1.00 & Ref. & 1.00 & Ref. & 1.00 & Ref. & 1.00 & Ref. & 1.00 & Ref. \\
\hline Q2 & 0.99 & $0.88,1.12$ & 1.06 & $0.94,1.20$ & 1.01 & $0.86,1.19$ & 1.09 & $0.86,1.38$ & 1.04 & $0.75,1.46$ \\
\hline Q3 & 1.00 & $0.89,1.13$ & $1 \cdot 13$ & $1.00,1.28$ & 1.04 & $0.88,1.23$ & 1.09 & $0.84,1.41$ & 1.65 & $1 \cdot 15,2 \cdot 36$ \\
\hline Q4 & $1 \cdot 10$ & $0.98,1.24$ & $1 \cdot 15$ & $1.02,1.30$ & 1.28 & $1.08,1.52$ & $1 \cdot 12$ & $0.84,1.49$ & 1.09 & $0.73,1.65$ \\
\hline Q5 (high adherence) & 1.13 & $1.00,1.28$ & 1.30 & $1.15,1.48$ & 1.41 & $1.17,1.70$ & 1.33 & $0.99,1.80$ & 1.86 & $1.18,2.95$ \\
\hline$P_{\text {trend }} \dagger$ & \multicolumn{2}{|c|}{0.005} & \multicolumn{2}{|c|}{$<0.001$} & \multicolumn{2}{|c|}{$<0.001$} & \multicolumn{2}{|c|}{0.096} & \multicolumn{2}{|c|}{0.019} \\
\hline
\end{tabular}

Ref., referent values.

* Adjusted models included age (continuous) at baseline, alcohol intake at baseline, race/ethnicity, annual family income, physical activity at baseline (continuous), smoking status at baseline and energy intake (continuous) at baseline. All non-continuous variables were modeled using disjoint indicator variables.

$\dagger P_{\text {trend }}$ corresponds to a Wald test statistic when a linear term for quintile of diet pattern was substituted in the model. 
further stratified their analyses to sub-classify individuals with obesity into class I, II or III. Moreover, our observation that no diet was protective against weight gain among those with a baseline $B M I \geq 35.0 \mathrm{~kg} / \mathrm{m}^{2}$ would suggest the need for intervention in these individuals before their progression from class I to class II obesity. Future studies are needed to identify the point at which this transition occurs in order to inform such intervention efforts

There are several limitations to our approach that warrant mention. Foremost, it should be noted that our sample comprised women who were predominantly non-Hispanic White (85.1\%), and thus findings may not be generalisable to minority populations. Second, although we found measured weight at baseline to be highly correlated with highest reported weight since last follow-up at year 1 , it has been previously shown that selfreported weight is prone to reporting error, and the magnitude and direction with which individuals misreport may vary by sex, age and weight status ${ }^{(33,34)}$. In addition, an epidemiological approach may have missed important confounding variables between dietary intake and weight gain. Additional limitations include the use of FFQ data to characterise diet and self-reported body weight, as measured weight was only available at two time points. Measurement error in diet assessment may have attenuated the relationship between diet and weight gain in our sample $e^{(35,36)}$. However, FFQ are better at capturing 'usual' diet than other transient methods (e.g. 24-h recall, food record, etc.) ${ }^{(37)}$, and intake from FFQ tend to be stable over time ${ }^{(37)}$. Thus, FFQ are well-suited for our study, in which greater within-person diet class stability over time would enhance our ability to examine the relationship between diet and weight gain. Moreover, it has been previously shown that dietary intake from the WHI FFQ had acceptable correlations with dietary intake from food records ${ }^{(38)}$. The inclusion of covariates related to misreporting of intake via $\mathrm{FFQ}^{(39)}$, as well as total energy intake, may have minimised the influence of FFQ-related measurement error on our findings. Fourth, although each of the four diet patterns was characterised using distinct criteria, it was possible for individuals to fall into more than one diet pattern. Nonetheless, diet patterns were modeled separately, thereby eliminating the possibility for an individual to represent more than one diet pattern within a given model. Finally, we chose a weight gain threshold of $\geq 10 \%$ to characterise weight gain, as the majority of women in our study gained weight during the course of follow-up. In sensitivity analyses, in which we explored the use of $\geq 5 \%$ weight gain as the outcome, we observed a similar pattern of findings for all but the low-fat diet pattern, thereby suggesting a degree of robustness to our principal findings. Nonetheless, in adjusted models using the lower threshold for weight gain, the relationship between the low-fat diet and risk of weight gain was in the opposite direction of that which we observed in our primary analyses. Notably, the significance of this finding is not clear. A possible explanation is that, because most women in our sample gained weight over time, the lower threshold of $\geq 5 \%$ weight gain resulted in little heterogeneity in the risk of weight gain between high and low adherers of each diet pattern. If true, then cautious interpretation of these findings would be warranted.

Despite these limitations, this study addresses a gap in research regarding the relationship between diet and long-term weight change among free-living individuals. Unlike weight loss trials, wherein the goal is for subjects to consume fewer energy content than expended, this study provides an examination of the relationship between diet and long-term weight change when subjects were not asked to change their diets. Moreover, whereas most prior studies have focused on weight loss, our focus on prevention of weight gain provides a unique contribution to the literature. Our results address the question "which diet is optimal for weight maintenance among free-living postmenopausal women who follow a diet of their own choosing?' We found that a reduced-carbohydrate diet, high in fat and protein intake, was associated with reduced risk of weight gain in postmenopausal women overall, whereas a low-fat a low-fat diet was associated with increased risk of postmenopausal weight gain.

\section{Conclusion}

Consuming a reduced-carbohydrate diet, with moderate fat and high protein intake, may decrease the risk of weight gain in postmenopausal women. However, prevailing dietary recommendations call for limiting fat intake in order to promote optimal health and prevent chronic disease. Our findings therefore challenge prevailing dietary recommendations, suggesting instead that a lowfat may promote rather than prevent weight gain after menopause.

\section{Acknowledgements}

Funding for C. F. and S. C. comes from the National Institutes of Health, National Cancer Institute (5 R25 CA057730-24). The Women's Health Initiative programme is funded by the National Heart, Lung, and Blood Institute, National Institutes of Health, US Department of Health and Human Services through contracts HHSN268201100046C, HHSN26801100001C, HHSN 268201100002C, HHSN268201100003C, HHSN268201100004C and HHSN271201100004C.

C. F. designed the study, completed the analyses and drafted and revised the paper. He is the guarantor. S. C. and A. C. F.-W. provided oversight and guidance during the planning phase and were instrumental in writing the manuscript proposal. In addition, S. C. and A. C. F.-W. helped to interpret analytic results, and provided extensive review, edits and feedback on the manuscript. M. Z. V. and J. I. F. also contributed extensively to the manuscript proposal and manuscript by providing critical review, commentary and edits. B. V. H., J. J. R., M. S., B. C., L. S. and R. U. provided additional feedback on the manuscript proposal, as well as extensive feedback, edits and commentary on the manuscript through several rounds of internal revision before the manuscript's submission to the journal. B. V. H., L. S. and M. S. also had instrumental roles in developing, planning and implementing one or more components of the Women's Health Initiative Study.

The authors declare that there are no conflicts of interest.

\section{References}

1. Wing RR, Matthews KA, Kuller LH, et al. (1991) Weight gain at the time of menopause. Arch Intern Med 151, 97-102. 
2. Polotsky HN \& Polotsky AJ (2010) Metabolic implications of menopause. Semin Reprod Med 28, 426-434.

3. Masters RK, Reither EN, Powers DA, et al. (2013) The impact of obesity on US mortality levels: the importance of age and cohort factors in population estimates. Am J Public Health 103, 1895-1901.

4. Borrell LN \& Samuel L (2014) Body mass index categories and mortality risk in US adults: the effect of overweight and obesity on advancing death. Am I Public Health 104, 512-519.

5. Shai I, Schwarzfuchs D, Henkin Y, et al. (2008) Weight loss with a low-carbohydrate, Mediterranean, or low-fat diet. $N$ Engl J Med 359, 229-241.

6. Esposito K, Kastorini C-M, Panagiotakos DB, et al. (2011) Mediterranean diet and weight loss: meta-analysis of randomized controlled trials. Metab Syndr Relat Disord 9 , 1-12.

7. Pelkman CL, Fishell VK, Maddox DH, et al. (2004) Effects of moderate-fat (from monounsaturated fat) and low-fat weightloss diets on the serum lipid profile in overweight and obese men and women. Am J Clin Nutr 79, 204-212.

8. Howard BV, Manson JE, Stefanick ML, et al. (2006) Low-fat dietary pattern and weight change over 7 years: the Women's Health Initiative Dietary Modification Trial. JAMA 295 , 39-49.

9. Avenell A, Brown T, McGee M, et al. (2004) What are the longterm benefits of weight reducing diets in adults? A systematic review of randomized controlled trials. J Hum Nutr Diet $\mathbf{1 7}$, 317-335.

10. Study TWsHI (1998) Design of the Women's Health Initiative clinical trial and observational study. Control Clin Trials 19 61-109.

11. Schakel S, Sievert Y \& Buzzard I (1988) Sources of data for developing and maintaining a nutrient database. I Am Diet Assoc 88, 1268-1271.

12. Kristal AR, Shattuck AL \& Williams AE (1992) Food frequency questionnaires for diet intervention research. In Proceedings of the 17th National Nutrient Databank Conference, June 1992, Washington, DC, pp. 110-125.

13. Guenther PM, Casavale KO, Reedy J, et al. (2013) Update of the healthy eating index: HEI-2010. J Acad Nutr Diet 113 , 569-580.

14. Bowman SA, Friday JE \& Moshfegh AJ (2008) MyPyramid Equivalents Database, 2.0 for USDA Survey Foods, 20032004: Documentation and User Guide. Beltsville, MD: Food Surveys Research Group, US Department of Agriculture, Beltsville Human Nutrition Research Center, Agricultural Research Service.

15. Fung TT, McCullough ML, Newby P, et al. (2005) Diet-quality scores and plasma concentrations of markers of inflammation and endothelial dysfunction. Am J Clin Nutr 82, 163-173.

16. Meyer AM, Evenson KR, Morimoto L, et al. (2009) Test-retest reliability of the Women's Health Initiative physical activity questionnaire. Med Sci Sports Exerc 41, 530-538.

17. Neuhouser ML, Di C, Tinker LF, et al. (2013) Physical activity assessment: biomarkers and self-report of activity-related energy expenditure in the WHI. Am $J$ Epidemiol 177, 576-585.

18. Pettee Gabriel K, McClain JJ, Lee CD, et al. (2009) Evaluation of physical activity measures used in middle-aged women. Med Sci Sports Exerc 41, 1403-1412.

19. Allison PD (1982) Discrete-time methods for the analysis of event histories. Sociol Methodol 13, 61-98.

20. Willett WC, Howe GR \& Kushi LH (1997) Adjustment for total energy intake in epidemiologic studies. Am J Clin Nutr $\mathbf{6 5}$, 1220S-1228S.
21. Mozaffarian D, Hao T, Rimm EB, et al. (2011) Changes in diet and lifestyle and long-term weight gain in women and men. $N$ Engl J Med 364, 2392-2404.

22. Belin RJ, Greenland P, Allison M, et al. (2011) Diet quality and the risk of cardiovascular disease: the Women's Health Initiative (WHI). Am J Clin Nutr 94, 49-57.

23. Beresford SA, Johnson KC, Ritenbaugh C, et al. (2006) Low-fat dietary pattern and risk of colorectal cancer: the Women's Health Initiative Randomized Controlled Dietary Modification Trial. JAMA 295, 643-654.

24. Mendez MA, Popkin BM, Jakszyn P, et al. (2006) Adherence to a Mediterranean diet is associated with reduced 3-year incidence of obesity. J Nutr 136, 2934-2938.

25. Sanchez-Villegas A, Bes-Rastrollo M, Martinez-Gonzalez M, et al. (2006) Adherence to a Mediterranean dietary pattern and weight gain in a follow-up study: the SUN cohort. Int J Obes (Lond) 30, 350-358.

26. Frazier-Wood AC, Kim J, Davis JS, et al. (2015) In crosssectional observations, dietary quality is not associated with CVD risk in women; in men the positive association is accounted for by BMI. Br J Nutr 113, 1244-1253.

27. Gardner CD, Kiazand A, Alhassan S, et al. (2007) Comparison of the Atkins, Zone, Ornish, and Learn diets for change in weight and related risk factors among overweight premenopausal women: the A to Z Weight Loss Study: a randomized trial. JAMA 297, 969-977.

28. Foster GD, Wyatt HR, Hill JO, et al. (2010) Weight and metabolic outcomes after 2 years on a low-carbohydrate versus low-fat diet: a randomized trial. Ann Inter Med $\mathbf{1 5 3}$, $147-157$

29. Sacks FM, Bray GA, Carey VJ, et al. (2009) Comparison of weight-loss diets with different compositions of fat, protein, and carbohydrates. $N$ Engl J Med 360, 859-873.

30. He K, Hu FB, Colditz GA, et al. (2004) Changes in intake of fruits and vegetables in relation to risk of obesity and weight gain among middle-aged women. Int J Obes Relat Metab Disord 28, 1569-1574.

31. Field AE, Willett WC, Lissner L, et al. (2007) Dietary fat and weight gain among Women in the Nurses' Health Study. Obesity (Silver Spring) 15, 967-976.

32. Williamson DF, Kahn HS, Remington PL, et al. (1990) The 10 -year incidence of overweight and major weight gain in us adults. Arch Intern Med 150, 665-672.

33. Villanueva EV (2001) The validity of self-reported weight in US adults: a population based cross-sectional study. BMC Public Health 1, 11.

34. Gorber SC, Tremblay M, Moher D, et al. (2007) A comparison of direct vs. self-report measures for assessing height, weight and body mass index: a systematic review. Obes Rev 8, 307-326.

35. Willett W \& Lenart E (2013) Reproducibility and validity of foodfrequency questionnaires. In Nutritional Epidemiology, pp. 96141 [W Willett, editor]. New York: Oxford University Press.

36. Hu FB, Rimm E, Smith-Warner SA, et al. (1999) Reproducibility and validity of dietary patterns assessed with a foodfrequency questionnaire. Am J Clin Nutr 69, 243-249.

37. Salvini S, Hunter DJ, Sampson L, et al. (1989) Food-based validation of a dietary questionnaire: the effects of weekto-week variation in food consumption. Int J Epidemiol 18, 858-867.

38. Patterson RE, Kristal AR, Tinker LF, et al. (1999) Measurement characteristics of the Women's Health Initiative food frequency questionnaire. Ann Epidemiol 9, 178-187.

39. Horner NK, Patterson RE, Neuhouser ML, et al. (2002) Participant characteristics associated with errors in self-reported energy intake from the Women's Health Initiative foodfrequency questionnaire. Am J Clin Nutr 76, 766-773. 